\title{
Antifouling Polyhydroxysterols Isolated from a Palauan Octocoral of Sinularia sp.
}

\author{
Shigeyuki Mizobuchi, ${ }^{\dagger}$ Kyoko Adachi, \\ and Wataru Miki ${ }^{\dagger \dagger}$ \\ Marine Biotechnology Institute (MBI), Shimizu Laboratories, \\ Sodeshi, Shimizu, Shizuoka 424, Japan
}

(Received March 31, 1995)

In our continuing search for effective antifoulants from marine invertebrates which can be used as substitutes for effective but highly toxic agents such as organotin compounds, we found that the lipophilic fraction from the extract of a Palauan octocoral of Sinularia sp. inhibited settlement of the blue mussel Mytilus edulis. Bioassay-guided isolation afforded five polyhydroxylated sterols with or without a monoacetyl functional group such as $6 \beta$-acetoxy-24-methylenecholestane- $3 \beta, 5 \alpha$-diol. The inhibitory activity of one of these compounds against the settlement of the blue mussel was found to be $34 \%$ of that of $\mathrm{CuSO}_{4}$, a standard antifouling agent. The activity of all five compounds was approximately 2 to 5 times stronger than that of cholesterol, $\beta$-cholestanol and $\beta$-sitosterol, suggesting the importance of the hydroxyl and/or acetoxyl groups for antifouling activity.

Key words: Mytilus edulis, antifoulants, $6 \beta$-acetoxy-24-methylenecholestane- $3 \beta, 5 \alpha$-diol, trihydroxylated steroids, octocoral, Sinularia sp.

Marine fouling organisms such as barnacles and mussels cause serious problems to ship hulls, cooling systems of power plants, aquaculture systems, and other man-made submersible structures. Organotin compounds such as TBTO [bis-( $n$-tributyltin) oxide] are currently used as antifoulants and are effective for preventing the settlement of sessile invertebrates. However, their toxic effects on fishes and shellfishes have caused serious concern, therefore nonor less-toxic substitutes for these organometallic chemicals are urgently required. We have been researching chemical signals, such as repellents and attractants, among marine invertebrates, and isolated several antifoulants from marine invertebrates. ${ }^{1-5)}$ The bioassay methods used for assessing their settlement inhibitory activities against fouling invertebrates have been applied to the blue mussel Mytilus edulis, ${ }^{6}$ and cyprid larvae of the reared barnacle Balanus amphitrite." Among the isolated antifoulants, common steroids such as cholesterol, $\beta$-cholestanol and $\beta$-sitosterol showed weak inhibitory activity against the settlement of M. edulis. ${ }^{1)}$ In order to clarify the antifouling mechanism of sterols, we started to search for steroidal antifoulants from marine invertebrates. During this study, we were able to isolate several polar steroids containing an acetoxy group from a marine octocoral Sinularia sp.

In this report, we describe the isolation, identification and inhibitory activity of these compounds.

\section{Materials and Methods}

\section{General Procedure}

${ }^{1} \mathrm{H}$ - and ${ }^{13} \mathrm{C}-\mathrm{NMR}$ spectra were recorded on a Varian Unity 500 NMR Spectrometer in $\mathrm{CDCl}_{3}$. Optical rotations were determined with a JASCO DIP-140 digital polarimeter at $24^{\circ} \mathrm{C}$, in $\mathrm{CHCl}_{3}$. Electron impact mass spectra (EIMS) and high-resolution electron impact mass spectra (HREIMS) were obtained with a JEOL JMS-SX102 mass spectrometer. Thin layer chromatography (TLC) was performed on silica gel 60 pre-coated plates (Merck) and spots were visualized by spraying with a $2 \%$ ceric sulfate solution in 5\% sulfuric acid and then heating on a hot plate.

The bioassay was carried out according to the method of Ina et al., ${ }^{,}$using the blue mussel M. edulis.

\section{Preparation of Extracts of Marine Invertebrates for the Bioassay}

Marine invertebrates, mainly sponges and octocorals, were collected around the palau Islands between October and November in 1991 by scuba diving during the cruise of the R/V Sogen Maru. Approximately 100 specimens were collected, stored in a freezer, and transported to our laboratory. Each frozen specimen $(100 \mathrm{~g})$ was soaked in acetone $(100 \mathrm{~m} l)$; the acetone extract was concentrated under reduced pressure, and partitioned between ethyl acetate and distilled water. Only the organic layer was subjected to the mussel assay.

\section{Isolation of the Active Compounds from Palauan Sinular- ia $s p$.}

Among one hundred invertebrate samples tested, the lipophilic layer from the extract of an octocoral of Sinular$i a$ sp. inhibited the settlement of $M$. edulis. Colonies of the soft coral were extracted with acetone whose extract gave the residue $(650 \mathrm{~g})$. This was partitioned between $\mathrm{CH}_{2} \mathrm{Cl}_{2}$

\footnotetext{
${ }^{\dagger}$ Applied Bioresearch Center, Kirin Brewery Co. Ltd., Miyahara, Takasaki, Gunma 370-12, Japan.

it Suntory Institute for Fundamental Research, Wakayamadai, Shimamoto, Mishima, Osaka 618, Japan.
} 


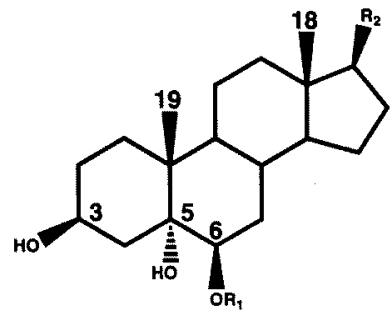

1: $\mathrm{R}_{1}=\mathrm{CH}_{3} \mathrm{CO} \quad \mathrm{R}_{2}={ }^{21}$

3: $\mathrm{R}_{1}=\mathrm{CH}_{3} \mathrm{CO} \quad \mathrm{R}_{2}=$ "Is"

4: $\mathrm{R}_{1}=\mathrm{CH}_{3} \mathrm{CO} \quad \mathrm{R}_{2}=\mathrm{M}_{\mathrm{s}}$

5: $\quad \mathbf{R}_{\mathbf{1}}=\mathbf{H}$

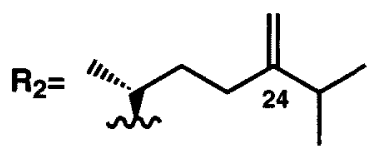

Fig. 1. Structures of antifouling steroids isolated from a Palauan octocoral Sinularia sp.

and $\mathrm{H}_{2} \mathrm{O}$. The organic layer $(6 \mathrm{~g})$ was chromatographed on a silica gel column (Silica gel 60, 230-400 mesh, Merck) with $\mathrm{CHCl}_{3} / \mathrm{MeOH}$ systems. Fraction I $(500 \mathrm{mg})$ eluted with $\mathrm{CHCl}_{3}$ was gelfiltered on a Sephadex $\mathrm{LH}-20$ with $\mathrm{CHCl}_{3}$ to give a colorless powder, which was then subjected to HPLC on an ODS column (Cosmosil $5 \mathrm{C}_{18} \mathrm{AR}$, $250 \times 4 \mathrm{~mm}$, Nacalai tesque) using $90 \%$ aqueous methanol to afford compounds 1 (34.3 mg), $2(3.5 \mathrm{mg}), 3(8.4 \mathrm{mg})$ and $4(19.1 \mathrm{mg})$. Fraction II $(73 \mathrm{mg})$ eluted with $\mathrm{CHCl}_{3} /$ $\mathrm{MeOH}(10: 1)$ was crystallized from methanol to give compound $5(29 \mathrm{mg})$.

\section{Resuits and Discussion}

\section{Structural Elucidation of the Active Compounds}

The physico-chemical and spectroscopic data for compounds 1 to 5 were as follows:

Compound 1. $\mathrm{Mp} 104.5-105.5^{\circ} \mathrm{C}\left(\mathrm{CHCl}_{3} / \mathrm{CH}_{3} \mathrm{OH}\right) ;[\alpha]_{D^{-}}$ $23.3^{\circ}$ ( $c$ 0.06, $\mathrm{CHCl}_{3}$ ); HRMS: $m / z 474.3741\left(\mathrm{C}_{30} \mathrm{H}_{50} \mathrm{O}_{4}\right.$, calcd. for 474.3709); MS: $m / z, 474\left(\mathrm{M}^{+}, 27 \%\right), 456\left(\mathrm{M}^{+}-\right.$ $18,12), 414\left(\mathrm{M}^{+}-\mathrm{AcOH}, 43\right), 43(00) ;{ }^{1} \mathrm{H}-\mathrm{NMR}(500 \mathrm{MHz}$, $\left.\mathrm{CDCl}_{3}\right) \delta: 0.69\left(18-\mathrm{CH}_{3}, s\right), 0.94\left(21-\mathrm{CH}_{3}, d, \mathrm{~J}=6.8 \mathrm{~Hz}\right)$, $1.020,1.022\left(26,27-\mathrm{CH}_{3}\right.$, each $\left.d, \mathrm{~J}=7.0 \mathrm{~Hz}\right), 1.15(19-$ $\left.\mathrm{CH}_{3}, s\right), 2.06\left(\mathrm{CH}_{3} \mathrm{CO}, s\right), 2.22(25-\mathrm{H}$, septet, $\mathrm{J}=7.0 \mathrm{~Hz})$, $4.08(3-\mathrm{CH} \alpha, m), 4.66,4.72\left(=\mathrm{CH}_{2}\right.$, each $1 \mathrm{H}$, each $\left.s\right)$,
$4.69(6-\mathrm{CH} \alpha, b r s) ;{ }^{13} \mathrm{C}-\mathrm{NMR}\left(125 \mathrm{MHz}, \mathrm{CDCl}_{3} \delta: 12.2(q\right.$ 18-C), 16.5 ( $q, 19-\mathrm{C}), 18.7$ ( $q, 21-\mathrm{C}), 21.1$ ( $t, 11-\mathrm{C}), 21.4$ $\left(q, \mathrm{C}^{*} \mathrm{H}_{3} \mathrm{CO}\right), 21.8,22.0$ (each $\left.q, 26-\mathrm{C}, 27-\mathrm{C}\right), 24.1(t$, 15-C), $28.2(t, 16-\mathrm{C}), 30.7(t, 2-\mathrm{C}), 30.8(d, 8-\mathrm{C}), 31.0(t$, 23-C), $31.4(t, 7-\mathrm{C}), 32.0(t, 1-\mathrm{C}), 33.8(d, 25-\mathrm{C}), 34.7(t$, 22-C), $35.8(d, 20-C), 38.5(s, 10-C), 39.9(t, 12-C), 40.5$ $(t, 4-C), 42.8(s, 13-C), 45.4(d, 9-C), 55.9(d, 14-C), 56.0$ $(d, 17-\mathrm{C}), 67.3(d, 3-\mathrm{C}), 75.3(s, 5-C), 76.1(d, 6-\mathrm{C}), 106.0$ $(t, 28-\mathrm{C}), 156.8(s, 24-\mathrm{C}), 170.1\left(\mathrm{~s}, \mathrm{CH}_{3} \mathrm{C}^{*} \mathrm{O}\right)$.

Compound 2. Mp 101.0-102.0 ${ }^{\circ} \mathrm{C}\left(\mathrm{CHCl}_{3} / \mathrm{CH}_{3} \mathrm{OH}\right) ;[\alpha]_{D^{-}}$ $51.3^{\circ}\left(c 0.39, \mathrm{CHCl}_{3}\right)$; HRMS: $m / z 474.3724\left(\mathrm{C}_{30} \mathrm{H}_{50} \mathrm{O}_{4}\right.$, calcd. for 474.3709); MS: $m / z 474\left(\mathrm{M}^{+}, 20 \%\right), 456\left(\mathrm{M}^{+}\right.$$18,17), 414\left(\mathrm{M}^{+}-\mathrm{AcOH}, 21\right), 69(100), 43(85) ;{ }^{1} \mathrm{H}-\mathrm{NMR}$ $\left(\mathrm{CDCl}_{3}\right) \delta: 0.695\left(18-\mathrm{CH}_{3}, s\right), 0.82,0.83\left(26,27-\mathrm{CH}_{3}\right.$, each $d, \mathrm{~J}=7.2 \mathrm{~Hz}), 0.91\left(28-\mathrm{CH}_{3}, d, \mathrm{~J}=6.7 \mathrm{~Hz}\right), 1.01\left(21-\mathrm{CH}_{3}\right.$, $d, \mathrm{~J}=6.7 \mathrm{~Hz}), 1.15\left(19-\mathrm{CH}_{3}, s\right), 2.06\left(\mathrm{CH}_{3} \mathrm{CO}, s\right), 4.08(3-$ $\mathrm{CH} \alpha, m), 4.70(6-\mathrm{CH} \alpha, b r s), 5.14-5.24$ (22-and 23-CH, $2 \mathrm{H}, m) ;{ }^{13} \mathrm{C}-\mathrm{NMR}\left(\mathrm{CDCl}_{3}\right) \delta: 12.4(q, 18-\mathrm{C}), 16.5(q, 19-$ C), 17.6 $(t, 28-\mathrm{C}), 19.7,20.0$ (each $q, 26-\mathrm{C}, 27-\mathrm{C}), 20.9$ (4, 21-C), $21.1(t, 11-\mathrm{C}), 21.4\left(q, \mathrm{C}^{*} \mathrm{H}_{3} \mathrm{CO}\right), 24.1(t, 15-\mathrm{C})$, $28.5(t, 16-\mathrm{C}), 30.7(t, 2-\mathrm{C}), 30.8(d, 8-\mathrm{C}), 31.4(t, 7-\mathrm{C})$, $32.0(t, 1-\mathrm{C}) 33.1(d, 25-\mathrm{C}), 38.5(\mathrm{~s}, 10-\mathrm{C}), 39.8(t, 12-\mathrm{C})$, $40.1(d, 20-C), 40.6(t, 4-C), 42.7(s, 13-C), 42.8(d, 24-C)$, $45.5(d, 9-\mathrm{C}), 56.0(d, 14-\mathrm{C}), 56.1(d, 17-\mathrm{C}), 67.3(d, 3-\mathrm{C})$, $75.3(s, 5-C), 76.1(d, 6-C), 131.8(d, 23-C), 135.7(d, 22-$ C), $170.2\left(s, \mathrm{CH}_{3} \mathrm{C}^{*} \mathrm{O}\right)$.

Compound 3. Mp 100.5-101.0 ${ }^{\circ} \mathrm{C}\left(\mathrm{CHCl}_{3} / \mathrm{CH}_{3} \mathrm{OH}\right) ;[\alpha]_{\mathrm{D}^{-}}$ $32.3^{\circ}$ ( c 0.13, $\mathrm{CHCl}_{3}$ ); HRMS: $m / z 402.3494\left[\mathrm{C}_{27} \mathrm{H}_{46} \mathrm{O}_{2}\right.$ for $\left(\mathrm{M}^{+}-\mathrm{AcOH}\right)$, calcd. for 402.3498$]$; $\mathrm{MS}: m / z 462\left(\mathrm{M}^{+}\right.$, $2 \%), 444\left(\mathrm{M}^{+}-18,8\right), 402\left(\mathrm{M}^{+}-\mathrm{AcOH}, 100\right), 43(95) .{ }^{1} \mathrm{H}-$ NMR $\left(\mathrm{CDCl}_{3}\right) \delta: 0.68\left(18-\mathrm{CH}_{3}, s\right), 0.860,0.862(26,27-$ $\mathrm{CH}_{3}$, each $\left.d, \mathrm{~J}=7.2 \mathrm{~Hz}\right), 0.90\left(21-\mathrm{CH}_{3}, d, \mathrm{~J}=6.7 \mathrm{~Hz}\right)$, $1.14\left(19-\mathrm{CH}_{3}, s\right), 2.06\left(\mathrm{CH}_{3} \mathrm{CO}, s\right), 4.08(3-\mathrm{CH} \alpha, m), 4.70$ $(6-\mathrm{CH} \alpha, b r s) ;{ }^{13} \mathrm{C}-\mathrm{NMR}\left(\mathrm{CDCl}_{3} \delta: 12.2(q, 18-\mathrm{C}), 16.5(q\right.$, 19-C), $18.7(q, 21-\mathrm{C}), 21.1(t, 11-\mathrm{C}), 21.4\left(q, \mathrm{C}^{*} \mathrm{H}_{3} \mathrm{CO}\right)$, $22.6,22.8$ (each $q, 26-\mathrm{C}, 27-\mathrm{C}), 23.9(t, 23-\mathrm{C}) 24.1(t, 15-$ C), $28.0(d, 25-C), 28.2(t, 16-C), 30.6(t, 2-C), 30.7(d, 8-$ C), $31.4(t, 7-C), 32.0(t, 1-C), 35.8(d, 20-C), 36.2(t, 22-$ C), $38.4(s, 10-C), 39.5(t, 24-C) .39 .9(t, 12-C), 40.5(t, 4-$ C), $42.7(s, 13-C), 45.4(d, 9-C), 55.9(d, 17-C), 56.2(d$, 14-C), $67.3(d, 3-C), 75.3(s, 5-C), 76.1(d, 6-C), 170.3(s$, $\left.\mathrm{CH}_{3} \mathrm{C}^{*} \mathrm{O}\right)$.

Compound 4. $\mathrm{Mp} 102.0-104.0^{\circ} \mathrm{C}\left(\mathrm{CHCl}_{3} / \mathrm{CH}_{3} \mathrm{OH}\right) ;[\alpha]_{\mathrm{D}^{-}}$ $26.3^{\circ}$ ( c 0.18, $\mathrm{CHCl}_{3}$ ); HRMS: $m / z 458.3778\left[\mathrm{C}_{30} \mathrm{H}_{50} \mathrm{O}_{3}\right.$ for $\left(\mathrm{M}^{+}-\mathrm{H}_{2} \mathrm{O}\right)$, calcd. for 458.3760]; MS: $m / z 476\left(\mathrm{M}^{+}\right.$, $2 \%), 458\left(\mathrm{M}^{+}-18,8\right), 416\left(\mathrm{M}^{+}-\mathrm{AcOH}, 100\right), 43(95) ;{ }^{1} \mathrm{H}-$ NMR $\left(\mathrm{CDCl}_{3}\right) \delta: 0.68\left(18-\mathrm{CH}_{3}, s\right), 0.780,0.780(26,27-$ $\mathrm{CH}_{3}$, each $\left.d, \mathrm{~J}=7.0 \mathrm{~Hz}\right), 0.85\left(28-\mathrm{CH}_{3}, d, \mathrm{~J}=6.7 \mathrm{~Hz}\right)$, $0.91\left(21-\mathrm{CH}_{3}, d, \mathrm{~J}=6.7 \mathrm{~Hz}\right), 1.14\left(19-\mathrm{CH}_{3}, s\right), 2.06$ $\left(\mathrm{CH}_{3} \mathrm{CO}, s\right), 4.08(3-\mathrm{CH} \alpha, m), 4.70(6-\mathrm{CH} \alpha, b r s) ;{ }^{13} \mathrm{C}-$ NMR $\left(\mathrm{CDCl}_{3}\right) \delta: 12.2(q, 18-\mathrm{C}), 15.5(q, 28-\mathrm{C}), 16.5(q$, 19-C), 17.6, 20.5 (each $q, 26-\mathrm{C}, 27-\mathrm{C}), 18.8(q, 21-\mathrm{C}), 21.1$ $(t, 11-\mathrm{C}), 21.4\left(q, \mathrm{C}^{*} \mathrm{H}_{3} \mathrm{CO}\right), 24.1(t, 15-\mathrm{C}), 28.1(t, 16-\mathrm{C})$, $30.6(t, 2-\mathrm{C}), 30.6(t, 23-\mathrm{C}), 30.7(d, 8-\mathrm{C}), 31.4(t, 7-\mathrm{C})$, $31.5(d, 25-\mathrm{C}), 32.0(t, 1-\mathrm{C}), 33.7(t, 22-\mathrm{C}), 36.2(d, 20-\mathrm{C})$, $38.5(s, 10-\mathrm{C}), 39.1(d, 24-\mathrm{C}), 39.9(t, 12-\mathrm{C}), 40.5(t, 4-\mathrm{C})$, $42.7(s, 13-\mathrm{C}), 45.4(d, 9-\mathrm{C}), 55.9(d, 17-\mathrm{C}), 56.1(d, 14-\mathrm{C})$, $67.3(d, 3-C), 75.3(s, 5-C), 76.0(d, 6-C), 170.3(s$, $\mathrm{CH}_{3} \mathrm{C}^{*} \mathrm{O}$ ).

Compound 5. Mp 223-224. $5^{\circ} \mathrm{C}\left(\mathrm{CHCl}_{3} / \mathrm{CH}_{3} \mathrm{OH}\right) ;[\alpha]_{D^{-}}$ $1.7^{\circ}$ (c $0.26, \mathrm{CHCl}_{3}$ ); HRMS: $m / z 432.3601\left(\mathrm{C}_{28} \mathrm{H}_{48} \mathrm{O}_{3}\right.$, calcd. for 432.3604$) ;{ }^{1} \mathrm{H}-\mathrm{NMR}\left(\mathrm{CDCl}_{3}\right) \delta: 0.68\left(18-\mathrm{CH}_{3}, s\right)$, 
$0.94\left(21-\mathrm{CH}_{3}, d, \mathrm{~J}=6.8 \mathrm{~Hz}\right), 1.020,1.022\left(26,27-\mathrm{CH}_{3}\right.$, each $d, \mathrm{~J}=7.0 \mathrm{~Hz}), 1.184\left(19-\mathrm{CH}_{3}, s\right), 2.22(25-\mathrm{H}$, septet, $\mathrm{J}=7.0 \mathrm{~Hz}), 3.54(6-\mathrm{CH} \alpha, b r s), 4.10(3-\mathrm{CH} \alpha, m), 4.66$, $4.72\left(=\mathrm{CH}_{2}\right.$, each $1 \mathrm{H}$, each $\left.s\right) ;{ }^{13} \mathrm{C}-\mathrm{NMR}\left(\mathrm{CDCl}_{3}\right) \delta: 12.2$ $(q, 18-\mathrm{C}), 16.9(q, 19-\mathrm{C}), 18.7(q, 21-\mathrm{C}), 21.2(t, 11-\mathrm{C})$, $21.8,22.0$ (each $q, 26-\mathrm{C}, 27-\mathrm{C}), 24.2(t, 15-\mathrm{C}), 28.2(t, 16-$ C), $30.2(d, 8-\mathrm{C}), 30.9(t, 2-\mathrm{C}), 31.1(t, 23-\mathrm{C}), 32.4(t, 1-\mathrm{C})$, $33.8(d, 25-C), 34.6(t, 7-C), 34.7(t, 22-C), 35.8(d, 20-C)$, $38.3(s, 10-\mathrm{C}), 40.0(t, 12-\mathrm{C}), 40.8(t, 4-\mathrm{C}), 42.8(s, 13-\mathrm{C})$, $45.9(d, 9-\mathrm{C}), 56.0(d, 14-\mathrm{C}), 56.1(d, 17-\mathrm{C}), 67.7(d, 3-\mathrm{C})$, $76.1(s, 5-C), 76.1(d, 6-C), 106.0(t, 28-C), 156.8(s, 24-C)$.

The data for compound 5 coincide well with those for 24-metylenecholestane- $3 \beta, 5 \alpha, 6 \beta$-triol. ${ }^{8)}$

On comparison of the NMR data of compound 1 with those of compound $\mathbf{5}$, they were closely related to each other, except for a signal due to an acetyl group at $\delta 2.06$ $(3 \mathrm{H}, s)$, and a low-field shift of the $6-\mathrm{CH} \alpha$ signal $\delta 4.69$ $(1 \mathrm{H}$, brs $)$ in compound 1 , while the latter appeared at $\delta$ $3.54(1 \mathrm{H}$, brs $)$ in compound 5 . These data indicate that compound 1 was the $6-O$-acetyl derivative of compound 5 , i.e. $6 \beta$-acetoxy-24-methylenecholestane- $3 \beta, 5 \alpha$-diol, which is found as a novel sterol with an acetyl group.

The 'H-NMR spectra for compounds 1-4 showed essentially the same chemical shifts for the 3-CH, 6-CH, 18-CH and $19-\mathrm{CH}$, indicating that they possessed the common steroidal nucleus with different side chain structures. The structures of the side chains were elucidated by comparing of the ${ }^{1} \mathrm{H}$ - and ${ }^{13} \mathrm{C}-\mathrm{NMR}$ data with those of known steroids. ${ }^{9-11)}$ The configuration of C-24 for compounds 2 and 4 was determined according to the method reported by Wright et al. ${ }^{11)}$ Thus, the structures of compounds 2,3 and 4 were established as $6 \beta$-acetoxy-24(R)-methylcholest-22ene- $3 \beta, 5 \alpha$-diol, $6 \beta$-acetoxy-cholestane- $3 \beta, 5 \alpha$-diol and $6 \beta$-acetoxy-24(S)-methylcholestane- $3 \beta, 5 \alpha$-diol, respectively. Compounds 1 and 4 were first isolated from the octocoral Sinularia dissecta by Bortolotto et al., ${ }^{12)}$ although both compounds were shown to be a mixture under chromatographic conditions. Compound 4 was also chemically synthesized by Pruna et al. ${ }^{8)}$ Compounds 1 to 4 are monoacetates of trihydroxy sterols, which are rare in marine organisms.

\section{Settlement inhibitory activities of compounds 1 to 5}

Compound 1 showed "+ +" activity" at a dose of 0.7 $\mathrm{mg} / \mathrm{cm}^{2}$ (34 Units; ${ }^{6)} 34 \%$ of the activity of $\mathrm{CuSO}_{4}$, which is a standard antifoulant). Compounds 2, 3 and 4 exhibited "+" activity" at a dose of $0.35 \mathrm{mg} / \mathrm{cm}^{2}$, while 5 had "+" activity at a dose of $1.0 \mathrm{mg} / \mathrm{cm}^{2}$. Although further examination was not possible owing to the limited samples, the acetyl group seems to play an important role in antifouling activity.

The activities of compounds 1 to 5 were approximately 2 to 5 times stronger than those of such conventional sterols as cholesterol, $\beta$-cholestanol and $\beta$-sitosterol, which were isolated by our group as antifoulants with weak activity, ${ }^{\text {l) }}$ again suggesting the importance of multiple hydroxyl and/or acetoxyl groups for the antifouling activity. These steroids might act as defense substances for preventing other organisms from settling.

Acknowledgments We thank Prof. K. Ina of Shizuoka University, and Prof. S. Miyachi, Drs. M. Endo and K. Kon-ya of MBI for valuable discussions. This work was performed as a part of the Industrial Science and Technology Frontier Program supported by the New Energy and Industrial Technology Development Organization.

\section{References}

1) S. Mizobuchi, N. Shimidzu, M. Katsuoka, K. Adachi, and W. Miki: Antifouling substances against the mussel in an octocoral Dendronephthya sp. Nippon Suisan Gakkaishi, 59, 1195-1199 (1993).

2) N. Shimidzu, M. Katsuoka, S. Mizobuchi, K. Ina, and W. Miki: Isolation of $(-)-\beta$-bisabolene from the octocoral Sinuiaria sp. as an effective repellent substance against the blue mussel, Mytilus edulis. Nippon Suisan Gakkaishi, 59, 1951 (1993).

3) M. Kawamata, K. Kon-ya, and W. Miki: Trigonelline, an antifouling substance isolated from an octocoral Dendronephthya sp. Fisheries Sci., 60, 485-486 (1994).

4) S. Mizobuchi, K. Kon-ya, K. Adachi. M. Sakai, and W. Miki: Antifouling substances from a Palauan octocoral Sinularia sp. Fisheries Sci., 60, 345-346 (1994).

5) K. Kon-ya, N. Shimidzu, K. Adachi, and W. Miki: 2,5,6-Tribromo1-methylgramine, an antifouling substance from the marine bryozoan Zoobotryon pellucidum. Fisheries Sci., 60, 773-775 (1994).

6) K. Ina, R. Takasawa, A. Yagi, N. Yamashita, H. Etoh, and K. Sakata: An improved assay method for antifouling substances using the blue mussel Mytilus edulis. Agric. Biol. Chem., 53, 33193321 (1989).

7) K. Kon-ya and W. Miki: All-seasonal assay for antifouling substances using reared barnacle larvae. J. Mar. Biotechnol., 1, 193195 (1994).

8) L. B. Pruna, R. D. Henriques, S. Huneck, K. Schreiber, and A. Preiss: Chemical studies of Cuban gorgoians. Part 10: Di- and trihydroxysteroids from Plexaurella grisea. Pharmazie, 39, 117-120 (1984).

9) A. Migliuolo, G. Notaro, V. Piccialli, and D. Sica: New tetrahydroxylated sterols from the marine sponge Spongia officinalis. $J$. Nat. Prod., 53, 1414-1424 (1990).

10) G. Notaro, V. Piccialli, D. Sica, and G. Corriero: $3 \beta, 5 \alpha, 6 \beta$-Trihydroxylated sterols with a saturated nucleus from two polulations of the marine sponge Cliona copisa. J. Nat. Prod., 54, 1570-1575 (1991).

11) J. L. C. Wright, A. G. McInnes, S. Shimizu, D. G. Smith, J. A. Walter, D. Idler, and W. Khalil: Identification of C-24 alkyl epimers of marine sterols by ${ }^{13} \mathrm{C}$ nuclear magnetic resonance spectroscopy. Can. J. Chem., 56, 1898-1903 (1978).

12) M. Bortolotto, J. C. Braekmen, D. Daloze, and B. Tursch: Chemical studies of marine invertebrates. XVIII. Four novel polyhydroxylated steroids from Sinularia dissecta (Coelenterata, Octocorallia, Alcyonacea). Bull. Soc. Chim. Belg., 85, 27-34 (1976). 